\title{
Nonthermal THz to TeV Emission from Stellar Wind Shocks in the Galactic Center
}

\section{Citation}

Quataert, Eliot, and Abraham Loeb. 2005. "Nonthermal THz to TeV Emission from Stellar Wind Shocks in the Galactic Center." The Astrophysical Journal 635 (1): L45-48. https:// doi.org/10.1086/499126.

\section{Permanent link}

http://nrs.harvard.edu/urn-3:HUL.InstRepos:41393439

\section{Terms of Use}

This article was downloaded from Harvard University's DASH repository, and is made available under the terms and conditions applicable to Other Posted Material, as set forth at http:// nrs.harvard.edu/urn-3:HUL.InstRepos:dash.current.terms-of-use\#LAA

\section{Share Your Story}

The Harvard community has made this article openly available.

Please share how this access benefits you. Submit a story.

Accessibility 


\title{
NONTHERMAL THz TO TeV EMISSION FROM STELLAR WIND SHOCKS IN THE GALACTIC CENTER
}

\author{
Eliot QuataerT ${ }^{1}$ AND Abraham LoeB ${ }^{2}$ \\ Received 2005 September 9; accepted 2005 October 26; published 2005 November 22
}

\begin{abstract}
The central parsec of the Galaxy contains dozens of massive stars with a cumulative mass-loss rate of $\sim 10^{-3}$ $M_{\odot} \mathrm{yr}^{-1}$. Shocks among these stellar winds produce the hot plasma that pervades the central part of the Galaxy. We argue that these stellar wind shocks also efficiently accelerate electrons and protons to relativistic energies. The relativistic electrons inverse Compton scatter the ambient ultraviolet and far-infrared radiation field, producing high-energy $\gamma$-rays with a roughly constant luminosity from $\sim \mathrm{GeV}$ to $\sim 10 \mathrm{TeV}$. This can account for the TeV source seen by HESS in the Galactic center. Our model predicts a GLAST counterpart to the HESS source with a luminosity of $\approx 10^{35} \mathrm{ergs}^{-1}$ and cooling break at $\approx 4 \mathrm{GeV}$. Synchrotron radiation from the same relativistic electrons should produce detectable emission at lower energies, with a surface brightness of $\approx 10^{-14} B_{-3}^{2} \mathrm{ergs} \mathrm{s}^{-1}$ $\mathrm{cm}^{-2} \operatorname{arcsec}^{-2}$ from $\sim \mathrm{THz}$ to $\sim \mathrm{keV}$, where $B_{-3}$ is the magnetic field strength in units of $\mathrm{mG}$. The observed level of diffuse thermal X-ray emission in the central parsec requires $B \lesssim 300 \mu \mathrm{G}$ in our models. Future detection of the diffuse synchrotron background in the central parsec can directly constrain the magnetic field strength, providing an important boundary condition for models of accretion onto Sgr A*.
\end{abstract}

Subject headings: Galaxy: center — radiation mechanisms: nonthermal — radiation mechanisms: thermal

\section{INTRODUCTION}

The young stellar cluster in the central parsec of the Galaxy contains nearly two dozen luminous blue supergiants and WolfRayet stars, in addition to a larger population of lower mass $\mathrm{O}$ and B stars (Krabbe et al. 1991; Genzel et al. 2003). These stars were produced in a short-lived compact starburst that occurred $\sim 10^{7}$ years ago. Collectively, the massive stars in the central parsec lose $\approx 10^{-3} M_{\odot} \mathrm{yr}^{-1}$ in stellar winds with velocities ranging from $\sim 300$ to $1000 \mathrm{~km} \mathrm{~s}^{-1}$ (Najarro et al. 1997; Paumard et al. 2001). Shocks produced by colliding stellar winds can account for the hot $(\approx 1-2 \mathrm{keV})$ X-ray-emitting plasma that fills the central few parsecs of the Galaxy (Quataert 2004; Rockefeller et al. 2004; Cuadra et al. 2005). These stellar winds are also the most likely source of fuel for the central massive black hole Sgr $\mathrm{A}^{*}: \approx 0.3 \%-1 \%$ of the mass lost to stellar winds is gravitationally captured by the black hole and flows in to smaller radii (e.g., Coker \& Melia 1997; Quataert 2004; Cuadra et al. 2005). The remaining mass is thermally driven out of the central stellar cluster in a wind.

In this Letter we investigate the possibility that stellar wind shocks in the Galactic center also efficiently accelerate electrons and protons to ultrarelativistic energies. This claim is motivated by the analogy between the strong collisionless shocks expected in the Galactic center and similar shocks in supernova remnants and $\gamma$-ray bursts that are inferred to be efficient sites of particle acceleration (e.g., Drury 1983; Blandford \& Eichler 1987). We show that a population of relativistic particles in the central parsec of the galaxy produces detectable emission from the radio to the $\gamma$-rays $(\S 2)$. We then compare the predictions of our model with current observations and discuss its further implications $(\S 3)$. We focus primarily on emission by relativistic electrons because it has fewer uncertainties, but we also briefly consider pion production by relativistic protons.

\footnotetext{
${ }^{1}$ Astronomy Department, 601 Campbell Hall, University of California, Berkeley, CA 94720; eliot@astron.berkeley.edu.

${ }^{2}$ Harvard-Smithsonian Center for Astrophysics, 60 Garden Street, Cambridge, MA 02138; aloeb@cfa.harvard.edu.
}

\section{PREDICTED SPECTRUM}

The total kinetic energy thermalized by shocks in the Galactic center is $\dot{E}=\frac{1}{2} f \dot{M}_{w} v_{w}^{2} \approx 10^{38} f_{0.3} \dot{M}_{-3} v_{8}^{2}$ ergs $\mathrm{s}^{-1}$, where $\dot{M}_{w}=10^{-3} \dot{M}_{-3} M_{\odot} \mathrm{yr}^{-1}$ is the total stellar mass-loss rate, $v_{w}=$ $1000 v_{8} \mathrm{~km} \mathrm{~s}^{-1}$ is the average wind speed, and $f=0.3 f_{0.3}$ accounts for geometric uncertainties such as the fraction of the stellar wind material that undergoes strong shocks (the normalization for $f$ is taken from the three-dimensional simulations of Rockefeller et al. 2004).

We assume that a fraction $\eta=10^{-2} \eta_{-2}$ of the shock energy goes into accelerating relativistic electrons, where the normalization of $\eta$ is motivated by observations of supernova remnants (see, e.g., § 2.2.2 of Keshet et al. 2003 for a summary of the relevant observations). Thus the energy supplied to relativistic electrons is

$$
\dot{E}_{e} \approx 10^{36} f_{0.3} \eta_{-2} \dot{M}_{-3} v_{8}^{2} \operatorname{ergs~s}^{-1} .
$$

We assume for simplicity that the electrons are injected with a spectrum $\propto \gamma^{-p}$ with $p=2$, similar to the index typically observed in supernova remnants (e.g., Brogan et al. 2005; Aharonian et al. 2005) and theoretically expected for strong shocks (Bell 1978; Blandford \& Ostriker 1978).

The high-energy electrons cool by synchrotron radiation and inverse Compton (IC) emission. Figure 1 summarizes our predictions for the spectrum produced by these two processes; the main features in this spectrum are described analytically below. The magnetic field in the central parsec is poorly constrained. We normalize our estimates using $B=B_{-3} \mathrm{mG}$, which is equipartition with the hot thermal plasma and is similar to the field strength inferred using the properties of nonthermal radio filaments on larger scales in the Galactic center (e.g., Morris \& Serabyn 1996; see, however, LaRosa et al. 2005). Equipartition fields could in principle also be generated by the shocks themselves (e.g., Medvedev \& Loeb 1999).

The young stellar cluster in the central parsec has an ultraviolet (UV) luminosity of $L=10^{41} L_{41} \mathrm{ergs} \mathrm{s}^{-1}$ within a radius $R=0.5 R_{0.5}$ pc (Krabbe et al. 1991), implying a radiation energy density of $U_{\mathrm{ph}}=10^{-7} U_{-7}^{\mathrm{UV}} \mathrm{ergs} \mathrm{cm}^{-3}$ with a characteristic 


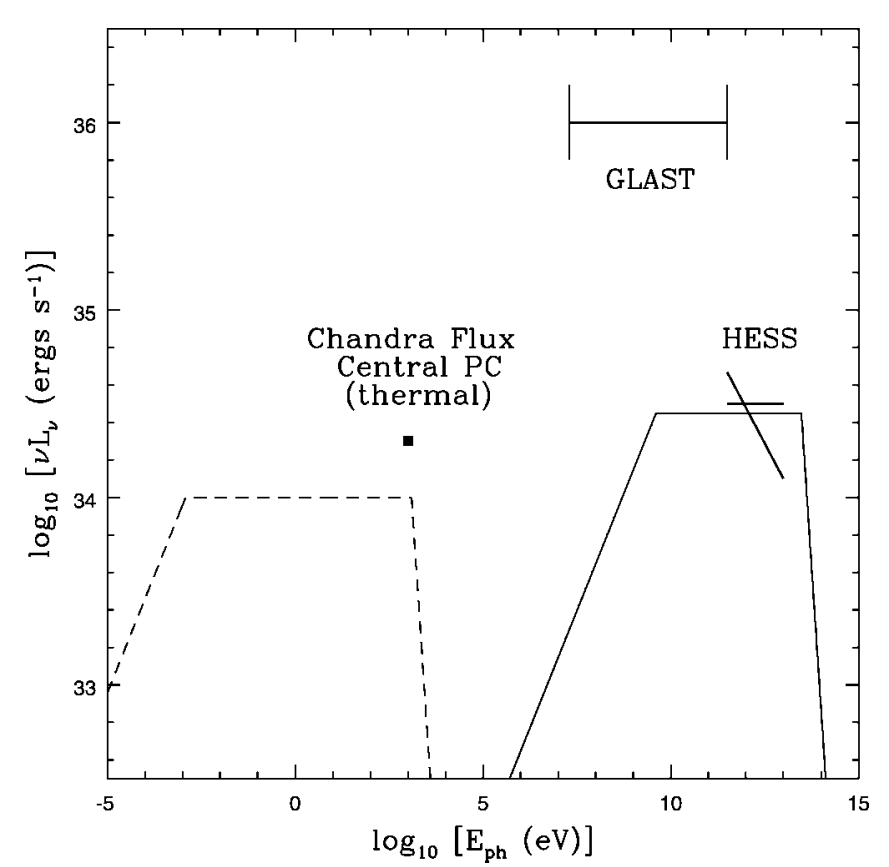

FIG. 1.-Predicted emission from shock-accelerated electrons in the central parsec of the Galactic center. The solid line shows the IC radiation (eq. [3]), while the dashed line shows the synchrotron radiation for $B=0.3 \mathrm{mG}$ and $U_{\text {ph }}=10^{-8}$ ergs $\mathrm{cm}^{-3}$ (eq. [8]). The parameter $\eta$ measuring the fraction of energy in shock-accelerated electrons is adjusted to reproduce the HESS TeV luminosity $\left(\eta \approx 5 \times 10^{-3}\right)$. With this normalization the $\gamma$-ray spectrum is uniquely determined, including the predicted cooling break in the GLAST band at $\approx 4 \mathrm{GeV}$; the flux of the synchrotron radiation depends primarily on the magnetic field strength in the central parsec. The high-energy cutoffs to the IC and synchrotron radiation are determined by $\gamma_{\max }$, the maximum Lorentz factor of accelerated electrons (eq. [4]). Klein-Nishina suppression of the electron scattering cross section produces a high-energy cutoff to the IC emission at comparable energies (eq. [7]). The exact spectral shape above the highenergy cutoff is uncertain and depends on the details of the electron distribution around $\gamma_{\max }$. The two power laws shown for the HESS data indicate the uncertainties in the power-law slope (Aharonian et al. 2004).

photon energy of $E_{\mathrm{ph}} \approx 10 \mathrm{eV}$. Only $\approx 20 \%$ of the UV radiation is absorbed by dust in situ (Davidson et al. 1992), which leads to a far-infrared (FIR) radiation field with $E_{\mathrm{ph}} \approx 0.04 \mathrm{eV}$ and $U_{-7}^{\mathrm{IR}} \sim 0.1$ or equivalently $U_{-8}^{\mathrm{IR}} \sim 1$.

The relativistic particles in the Galactic center are advected out of the central parsec with the thermally driven wind on a timescale $t_{\exp } \approx R / v_{w} \approx 500 R_{0.5} / v_{8}$ yr. Sufficiently relativistic electrons with $\gamma>\gamma_{c}$ cool on a timescale $\lesssim t_{\text {exp }}$. For our fiducial normalization the cooling is primarily by IC radiation (unless $B \gtrsim 1.6 \mathrm{mG}$ ). In this case the critical Lorentz factor where the IC cooling time equals the expansion time is given by

$$
\gamma_{c} \approx \frac{3 \pi m_{e} c^{2} R v_{w}}{\sigma_{\mathrm{T}} L} \approx 2 \times 10^{4} \frac{R_{0.5} v_{8}}{L_{41}}
$$

where $\sigma_{\mathrm{T}}$ is the Thompson cross section. Electrons with $\gamma>\gamma_{c}$ lose all of their energy to IC radiation before escaping the central parsec, producing a power

$$
\left.\nu L_{\nu}\right|_{\mathrm{IC}} \approx \frac{\dot{E}_{e}}{\ln \left(\gamma_{\max }\right)} \approx 6 \times 10^{34} f_{0.3} \eta_{-2} \dot{M}_{-3} v_{8}^{2} \operatorname{ergs~s}^{-1}
$$

where $\gamma_{\max }$ is the maximum Lorentz factor of the accelerated electrons. The exact value of $\gamma_{\max }$ is uncertain but a rough estimate can be obtained by balancing the cooling time with the acceleration time at the shock front, $t_{\mathrm{acc}} \approx \Omega^{-1} c^{2} / v_{w}^{2}$, where
$\Omega$ is the relativistic cyclotron frequency (e.g., Loeb \& Waxman 2000). This yields

$$
\gamma_{\max } \sim\left(\frac{e B}{\sigma_{\mathrm{T}} U_{\mathrm{ph}}}\right)^{1 / 2} \frac{v_{w}}{c} \sim 3 \times 10^{7} \frac{B_{-3}^{1 / 2} v_{8}}{\sqrt{U_{-8}^{\mathrm{IR}}}} .
$$

Note that in the above numerical estimate we have normalized the photon energy density using the FIR radiation field, while in our estimate of $\gamma_{c}$ in equation (2) we used the full UV radiation field. This is because electrons with $\gamma \sim \gamma_{\max }$ see UV photons in their rest frame as $\gamma$-rays with energy $\gg m_{e} c^{2}$ and do not efficiently scatter the UV photons due to the KleinNishina suppression of the cross section.

Equations (2) and (4) imply that the IC power in equation (3) applies for photon energies $E_{\min }<E<E_{\max }$, where

$$
E_{\mathrm{min}} \approx \gamma_{c}^{2} E_{\mathrm{ph}} \approx 4 \mathrm{GeV}\left(\frac{R_{0.5} v_{8}}{L_{41}}\right)^{2}\left(\frac{E_{\mathrm{ph}}}{10 \mathrm{eV}}\right)
$$

and

$$
E_{\mathrm{max}} \approx \gamma_{\mathrm{max}}^{2} E_{\mathrm{ph}} \approx 30 \mathrm{TeV}\left(\frac{B_{-3} v_{8}^{2}}{U_{-8}^{\mathrm{IR}}}\right)\left(\frac{E_{\mathrm{ph}}}{0.04 \mathrm{eV}}\right)
$$

Relativistic corrections to the electron scattering cross section lead to an additional suppression of the IC power above

$$
E_{b} \approx 25 \mathrm{TeV}\left(\frac{E_{\mathrm{ph}}}{0.04 \mathrm{eV}}\right)^{-1}
$$

For $E<E_{\min }$, the electrons do not lose all of their energy before flowing out of the central parsec and so the IC power is suppressed, with $\nu L_{\nu} \propto\left(E / E_{\min }\right)^{1 / 2}$.

\subsection{Synchrotron Emission}

The same electrons that produce IC power in the $\gamma$-rays will also produce synchrotron emission at lower energies, with $P_{s} / P_{\mathrm{IC}}=U_{B} / U_{\mathrm{ph}}$. For $\nu_{c}<\nu<\nu_{\max }$ the total synchrotron power is

$$
\left.\nu L_{\nu}\right|_{s} \approx 2 \times 10^{34}\left(\frac{B_{-3}^{2}}{U_{-7}^{\mathrm{UV}}}\right) f_{0.3} \eta_{-2} \dot{M}_{-3} v_{8}^{2} \operatorname{ergs~s}^{-1}
$$

where

$$
\nu_{c} \approx \frac{\gamma_{c}^{2} e B}{2 \pi m_{e} c} \approx 1 B_{-3}\left(\frac{R_{0.5} v_{8}}{L_{41}}\right)^{2} \mathrm{THz}
$$

and

$$
\nu_{\max } \approx \frac{\gamma_{\max }^{2} e B}{2 \pi m_{e} c} \approx 3 \times 10^{18}\left(\frac{B_{-3}^{2} v_{8}^{2}}{U_{-8}^{\mathrm{IR}}}\right) \mathrm{Hz} .
$$

Near $\nu_{\max }$ the electrons only IC scatter FIR photons and so $U_{\mathrm{ph}} \approx U_{-8}^{\mathrm{IR}}$ should be used in equation (8). Note that equation (8) assumes $U_{B}<U_{\mathrm{ph}}$; if $U_{B}>U_{\mathrm{ph}}$ the electrons lose their energy to synchrotron radiation, not IC scattering, and the total synchrotron power would be given by equation (3) instead of equation (8); as we show in the next section, however, $B$ must be $\leqslant 300 \mu \mathrm{G}$ in the context of our model, so this possibility is not realized.

Equations (8)-(10) imply that synchrotron emission from 
shock-accelerated electrons produces a diffuse radiation field from the radio to the X-rays in the central parsec. This emission would be extended on a radial scale of $\approx 0.5 R_{0.5} \mathrm{pc} \approx 10^{\prime \prime}$ and so would have a surface brightness of (for a distance to the Galactic center of $8 \mathrm{kpc}$ )

$$
I_{s} \approx 9 \times 10^{-15}\left(\frac{B_{-3}^{2}}{L_{41}}\right) f_{0.3} \eta_{-2} \dot{M}_{-3} v_{8}^{2} \operatorname{ergs~s}^{-1} \mathrm{~cm}^{-2} \operatorname{arcsec}^{-2} \text {. }
$$

\section{2. $\gamma$-Ray Emission from Pion Decay}

Relativistic protons can also be a significant source of highenergy emission through the production of neutral and charged pions; here we briefly estimate the expected $\gamma$-ray luminosity from neutral pion production in the central few parsecs of the Galaxy. We assume that a larger fraction, $\sim 10 \%$, of the shock energy goes into accelerating relativistic protons (Blandford \& Eichler 1987), in which case

$$
\dot{E}_{p} \approx 10^{37} f_{0.3} \eta_{-1} \dot{M}_{-3} v_{8}^{2} \operatorname{ergs~s}^{-1}
$$

The hot thermal plasma in the Galactic center has a density of $n \approx 30 \mathrm{~cm}^{-3}$ (e.g., Baganoff et al. 2003). The timescale for relativistic protons to lose their energy via neutral pion production is $t_{\text {pion }} \approx 10^{8} n^{-1}$ yr. Comparing this to the expansion time of the hot plasma of $\sim 500 \mathrm{yr}$, we find a very low $\mathrm{GeV}$ $\gamma$-ray luminosity from pion production on the ambient hot plasma, $\nu L_{\nu} \approx 10^{-4} \dot{E}_{p} / \ln \left(\gamma_{\max }\right) \sim 10^{32} \mathrm{ergs} \mathrm{s}^{-1}$.

A more promising source of $\gamma$-rays from pion decay in the central few parsecs is the interaction between the shockaccelerated protons and the circumnuclear disk (CND). The $\mathrm{CND}$ is a ring of dense molecular gas located $\approx 1.5 \mathrm{pc}$ from Sgr A*, with a scale height of $\sim 0.5$ pc (Jackson et al. 1993; Christopher et al. 2005). The mean density of the CND is at least $n \approx 10^{5} n_{5} \mathrm{~cm}^{-3}$, and Christopher et al. (2005) argue that it could be several orders of magnitude higher. The morphology of the diffuse X-ray emission in the central parsec suggests that the outflowing wind from the central star cluster impinges on and is confined by the CND (Baganoff et al. 2003; Rockefeller et al. 2004).

To estimate the $\gamma$-ray luminosity from pion decay in the CND, we assume that a fraction $\xi=0.1 \xi_{0.1}$ of the relativistic protons created in the central $0.5 \mathrm{pc}$ enter into the CND, and that a fraction $\sim \frac{1}{3}$ of the proton energy goes into $\gamma$-rays, rather than neutrinos or other particles. The normalization for $\xi$ is based on the solid angle subtended by the inner edge of the $\mathrm{CND}$, but in principle $\xi$ could be much smaller if relativistic particles are magnetically shielded out of the CND. The cooling time for relativistic protons due to pion production in the CND is $t_{\text {pion }} \approx 10^{3} n_{5}^{-1}$ yr. By contrast, the timescale for relativistic protons to escape from the CND is uncertain and depends sensitively on the magnetic field geometry and cross-field diffusion. Unless the effective diffusion velocity is $\gtrsim 1000 \mathrm{~km} \mathrm{~s}^{-1}$, however, the protons will lose all of their energy in the CND because the cooling time is shorter the escape time. Under this assumption, the $\gamma$-ray luminosity from the CND is

$$
\nu L_{\nu} \sim \frac{\xi \dot{E}_{p}}{3 \ln \left(\gamma_{\max }\right)} \sim 2 \times 10^{34} f_{0.3} \xi_{0.1} \eta_{-1} \dot{M}_{-3} v_{8}^{2} \operatorname{ergs~s}^{-1}
$$

Equation (13) predicts the maximum $\gamma$-ray luminosity not far below that produced by relativistic electrons (eq. [3]), but the estimate is much less certain because the relativistic protons might quickly leak out of the CND along open magnetic field lines. The spectrum of $\gamma$-rays from pion decay would extend from $\approx 70 \mathrm{MeV}$ to many $\mathrm{TeV}$, with the maximum photon energy determined by the maximum energy of the accelerated protons.

\section{DISCUSSION}

Figure 1 summarizes our estimates of the IC and synchrotron radiation expected from shock-accelerated electrons in the central parsec. We do not include the predicted radiation from pion decay estimated in $\S 2.2$ because it is much less certain, although we reiterate that the $\gamma$-ray flux from pion decay in the CND could be nonnegligible.

Figure 1 also summarizes some of the data relevant to our predictions. In particular, IC upscattering of the ambient FIR radiation field can account for the TeV source seen by HESS (Aharonian et al. 2005) and CANGAROO (Tsuchiya et al. 2004) toward the Galactic center. In Figure 1 we have adjusted the normalization of the IC component to account for the HESS luminosity by setting $\eta \approx 5 \times 10^{-3}$ in equation (3). Our model naturally accounts for the HESS spectrum and makes the strong prediction that the Gamma-Ray Large Area Space Telescope $(G L A S T)^{3}$ should observe a cooling break at $\approx 4 \mathrm{GeV}$. The energy of the cooling break is very well determined in our model (eq. [5]) since it is set by the UV radiation energy density and the expansion time of the thermal plasma, both of which are observationally well constrained.

Atoyan \& Dermer (2004) also explain the TeV emission from the Galactic center as IC scattering of FIR photons by relativistic electrons (see Pohl 1997 and Melia et al. 1998 for related ideas in the context of the EGRET source 3EG J1746-2851, which is now believed to be well offset from the central parsec; Pohl 2005). In their model, however, the electrons are produced in the termination shock of an outflow from Sgr A*. On energetic grounds, the shocked stellar winds investigated in this Letter may be a more important source of particle acceleration: the accretion rate onto $\operatorname{Sgr} \mathrm{A}^{*}$ is believed to be $\lesssim 10^{-8} M_{\odot} \mathrm{yr}^{-1}$ (e.g., Agol 2000; Quataert \& Gruzinov 2000), implying a total accretion power of $\approx 0.1 \dot{M} c^{2} \lesssim 5 \times 10^{37} \dot{M}_{-8} \mathrm{ergs} \mathrm{s}^{-1}$, somewhat smaller than the energy thermalized by shocked stellar winds in the central parsec. In addition, the presence of stellar wind shocks in the central parsec is observationally well established, while the properties of an outflow from Sgr A* (its mass-loss rate, collimation, etc.) are significantly less well understood.

In addition to IC emission at high energies, we also predict that synchrotron radiation from shock-accelerated electrons in the central parsec leads to a diffuse background of radiation from $\sim \mathrm{THz}$ to $\sim \mathrm{keV}$ (Fig. 1), with a surface brightness given by equation (11). This emission would be extended on the scale of the central stellar cluster, $\approx 10^{\prime \prime}$. The magnitude of the diffuse synchrotron emission is sensitive to the magnetic field strength in the central parsec, which is poorly constrained observationally. ${ }^{4}$ The most stringent observational limits to date on diffuse emission at the relevant wavelengths are probably from deep Chandra observations that find extended thermal emission with a surface brightness of $\approx 10^{-14} \mathrm{ergs} \mathrm{s} \mathrm{cm}^{-2} \operatorname{arcsec}^{-2}$ in the central few parsecs (Baganoff et al. 2003). Using equation (11) with $L_{41} \approx 0.1$, appropriate for the FIR radiation field scattered

\footnotetext{
${ }^{3}$ See http://glast.gsfc.nasa.gov.

${ }^{4}$ On much larger $\left(\gtrsim 2^{\prime}\right)$ scales, LaRosa et al. (2005) argue that the field strength is $\sim 10 \mu \mathrm{G}$, while the prevalence of nonthermal radio filaments has been used to argue for $B \sim \mathrm{mG}$ (e.g., Morris \& Serabyn 1996).
} 
by electrons with $\gamma \sim \gamma_{\max }$, we find that the Chandra observations require that the magnetic field strength in the central parsec be $\$ 300 \mu \mathrm{G}$ in order to not overproduce the extended $\mathrm{X}$-ray emission. It is worth highlighting that future probes of the nonthermal diffuse emission could directly constrain the magnetic field in the central parsec. In particular, the ratio of the luminosity of the diffuse emission to the luminosity of the HESS TeV source is determined by $U_{B} / U_{\mathrm{ph}}$ and thus can be used to measure $U_{B}$. Such a measurement would provide a very useful outer boundary condition for models of accretion onto Sgr A*, which are sensitive to the geometry and magnitude of the magnetic field at large radii (e.g., Igumenshchev \& Narayan 2002).

Lastly, we note that some fraction of the shock-accelerated electrons and protons in the central parsec should be gravitationally captured by $\mathrm{Sgr} \mathrm{A}^{*}$ along with the thermal plasma, providing a seed population of relativistic particles to the ac- cretion flow. The Lorentz factor of such particles will, at a minimum, increase via adiabatic compression as $\gamma \propto \rho^{1 / 3}$. The density contrast between the Bondi accretion radius $\left(R_{\mathrm{B}} \sim 10^{5}\right.$ Schwarzschild radii) and the event horizon is believed to be a factor of $\sim 10^{5}$ (see Quataert 2003 for a review), implying that mildly relativistic electrons at $\sim R_{B}$ have $\gamma \sim 100$ close to the black hole. Such electrons would emit in the millimeter to midIR and could contribute significantly to the observed emission from Sgr A*. This possibility will be explored in more detail in a future paper.

We thank Reinhard Genzel and Chuck Dermer for useful conversations. E. Q. thanks the ITC at Harvard for their hospitality. E. Q. is supported in part by NSF grant AST 02-06006, NASA grant NAG5-12043, an Alfred P. Sloan Fellowship, and the David and Lucile Packard Foundation. A. L. is supported in part by NASA grants NAG5-13292 and NNG05GH54G.

\section{REFERENCES}

Agol, E. 2000, ApJ, 538, L121

Aharonian, F., et al. 2004, A\&A, 425, L13 2005, A\&A, 437, L7

Atoyan, A., \& Dermer, C. D. 2004, ApJ, 617, L123

Baganoff, F. K., et al. 2003, ApJ, 591, 891

Bell, A. R. 1978, MNRAS, 182, 147

Blandford, R., \& Eichler, D. 1987, Phys. Rep., 154, 1

Blandford, R. D., \& Ostriker, J. P. 1978, ApJ, 221, L29

Brogan, C. L., Gaensler, B. M., Gelfand, J. D., Lazendic, J. S., Lazio, T. J. W., Kassim, N. E., \& McClure-Griffiths, N. M. 2005, ApJ, 629, L105

Christopher, M. H., Scoville, N. Z., Stolovy, S. R., \& Yun, M. S. 2005, ApJ, 622,346

Coker, R. F., \& Melia, F. 1997, ApJ, 488, L149

Cuadra, J., Nayakshin, S., Springel, V., \& Di Matteo, T. 2005, MNRAS, in press (astro-ph/0505382)

Davidson, J. A., Werner, M. W., Wu, X., Lester, D. F., Harvey, P. M., Joy, M., \& Morris, M. 1992, ApJ, 387, 189

Drury, L. O. 1983, Rep. Prog. Phys., 46, 973

Genzel, R., et al. 2003, ApJ, 594, 812

Igumenshchev, I. V., \& Narayan, R. 2002, ApJ, 566, 137
Jackson, J. M., Geis, N., Genzel, R., Harris, A. I., Madden, S., Poglitsch, A., Stacey, G. J., \& Townes, C. H. 1993, ApJ, 402, 173

Keshet, U., Waxman, E., Loeb, A., Springel, V., \& Hernquist, L. 2003, ApJ, 585,128

Krabbe, A., Genzel, R., Drapatz, S., \& Rotaciuc, V. 1991, ApJ, 382, L19

LaRosa, T. N., Brogan, C. L., Shore, S. N., Lazio, T. J., Kassim, N. E., \& Nord, M. E. 2005, ApJ, 626, L23

Loeb, A., \& Waxman, E. 2000, Nature, 405, 156

Medvedev, M. V., \& Loeb, A. 1999, ApJ, 526, 697

Melia, F., Yusef-Zadeh, F., \& Fatuzzo, M. 1998, ApJ, 508, 676

Morris, M., \& Serabyn, E. 1996, ARA\&A, 34, 645

Najarro, F., et al. 1997, A\&A, 325, 700

Paumard, T., Maillard, J. P., Morris, M., \& Rigaut, F. 2001, A\&A, 366, 466 Pohl, M. 1997, A\&A, 317, 441 2005, ApJ, 626, 174

Quataert, E. 2003, Astron. Nachr., 324 (Suppl. 1), 435 2004, ApJ, 613, 322

Quataert, E., \& Gruzinov, A. 2000, ApJ, 545, 842

Rockefeller, G., Fryer, C., Melia, F., \& Warren, M. 2004, ApJ, 604, 662

Tsuchiya, K., et al. 2004, ApJ, 606, L115 